\title{
«Actions en justice et rémunération des avocats : une revue de la littérature »
}

\author{
$\underline{\text { Auteurs }}$
}

Myriam Doriat-Duban, Yannick Gabuthy, Eve-Angéline Lambert

Document de Travail $n^{\circ} 2019-09$

Mars 2019

Bureau d'Économie

Théorique et Appliquée

BETA

www.beta-umr7522.fr

@beta_economics

Contact :

jaoulgrammare@beta-cnrs.unistra.fr 


\title{
Actions en justice et rémunération des avocats : une revue de la littérature
}

\author{
Myriam Doriat-Duban* $\quad$ Yannick Gabuthy ${ }^{\dagger} \quad$ Eve-Angéline Lambert ${ }^{\ddagger}$
}

Février 2019

\begin{abstract}
Résumé
Plusieurs pays européens ont désormais adopté (ou envisagent d'adopter) l'honoraire de résultat comme mécanisme de rémunération des avocats dans le cadre d'actions en responsabilité civile. Ce type d'honoraire implique que l'avocat ne soit rémunéré qu'en cas de transaction amiable ou de victoire au procès, percevant soit un bonus horaire (ou forfaitaire), soit une part de l'indemnisation obtenue par son client. Dans cet article, nous dressons un état des lieux de la littérature économique théorique portant sur l'analyse de ces modalités de rémunération, via l'étude de leurs implications sur les comportements des parties prenantes au litige (que ce soit lors de sa résolution ou, plus en amont, lors de son émergence). Sur cette base, il apparaît que l'honoraire de résultat présente plusieurs avantages potentiels, notamment en termes d'accès au droit, de sélection des affaires, de transfert de risque et de régulation du phénomène d'aléa moral, nous amenant à penser que sa généralisation à l'ensemble des pays occidentaux est souhaitable à de nombreux égards. Pour être plus affirmatif, il faudrait néanmoins que ces avantages soient plus largement confirmés sur un plan empirique.
\end{abstract}

Mots clés : Action en responsabilité civile, Règlement amiable et procès, Avocats, Honoraire de résultat.

Classification JEL : K13, K40, K41.

"Litigation does not present a single externality that might be corrected with a single policy tool. It presents a complex bundle of positive and negative externalities whose correction requires an array of policy instruments." Avery W. Katz et Chris W. Sanchirico, Fee Shifting in Litigation : Survey and Assessment, 2011.

*BETA (CNRS, Université de Strasbourg, Université de Lorraine) ; 13, place Carnot, C.O. 70026, 54035 Nancy, France. Email : myriam.duban@univ-lorraine.fr.

${ }^{\dagger}$ BETA (CNRS, Université de Strasbourg, Université de Lorraine) ; 13, place Carnot, C.O. 70026, 54035 Nancy, France. Email : yannick.gabuthy@univ-lorraine.fr.

${ }^{\ddagger}$ BETA (CNRS, Université de Strasbourg, Université de Lorraine) ; 13, place Carnot, C.O. 70026, 54035 Nancy, France. Email : eve-angeline.lambert@univ-lorraine.fr. 


\section{INTRODUCTION}

Une évaluation de l'activité judiciaire française en 2017 estime à 2677253 le nombre d'affaires nouvelles traitées par les juridictions civiles et commerciales. Ces affaires concernent principalement les tribunaux d'instance (24\%) et de grande instance (36\%), le reste se répartissant entre les cours d'appel, les conseils de prud'hommes et les tribunaux de commerce (Chambaz, 2017). Ces instances traitent notamment des litiges en responsabilité civile, recours qui sont consécutifs à un accident ou à une transaction commerciale : le demandeur, s'affirmant victime d'un préjudice occasionné par le défendeur, prétend faire reconnaître par voie de justice son droit à recevoir une compensation financière (dommages-intérêts) de la part de ce dernier. La procédure de règlement du différend se déroule alors potentiellement en trois temps, suivant l'issue de chaque étape : l'instant où la victime décide de poursuivre, ou non, son offenseur présumé; le moment où les parties entrent en négociation dans la perspective d'une éventuelle transaction amiable; le temps du procès à l'issue duquel un jugement est rendu par le tribunal. Dans ce type de contentieux, l'opportunité de faire appel à un avocat fait partie des droits des justiciables.

Jusqu'au début des années 1990, en Europe, le mode de rétribution des avocats dans le cadre d'une action en responsabilité civile était fondé sur une rémunération horaire (ou au temps passé) : l'honoraire était calculé en appliquant un taux de rémunération horaire au nombre d'heures effectuées par l'avocat. Cependant, s'inspirant de la pratique américaine, un certain nombre de pays, tels que le Royaume-Uni ou l'Australie, ont assez récemment adopté des systèmes d'honoraire conditionnel : l'avocat est rémunéré uniquement s'il obtient gain de cause en justice, via le versement de dommages-intérêts au procès ou l'obtention d'une transaction amiable, et perçoit ainsi une rémunération horaire à laquelle s'ajoute un bonus s'appliquant à chaque heure effectuée. ${ }^{1}$ La pratique américaine de rémunération contingente au résultat est légèrement différente puisque l'avocat ne perçoit pas de rétribution horaire, ni de bonus forfaitaire, mais une part des dommages-intérêts obtenus par son client au procès (ou à l'issue d'une transaction). En contrepartie, l'avocat est supposé assumer l'ensemble des coûts générés par la procédure judiciaire, que ce soit en cas d'honoraire contingent ou conditionnel. ${ }^{2}$ Sur cette base, l'objectif de cet article est de dresser un état des lieux de la littérature portant sur l'analyse économique de ces mécanismes de rémunération, qui sont appréhendés via leurs effets sur les comportements des différents protagonistes du litige (avocats, clients, et juges le cas échéant). Suivant les préceptes sous-jacents à l'économie du droit, en tant que champ d'analyse, la règle juridique ne peut être considérée comme une norme édictée par le politique dont l'application par l'agent suivrait un processus déterministe. ${ }^{3}$ La conceptualisation économique repose en effet sur l'idée que l'agent se saisit de la règle de droit, via un processus d'optimisation, afin d'en faire le support de son comportement,

1. Une forme alternative d'honoraire conditionnel existe également dans laquelle le bonus est forfaitaire, c'est-àdire non appliqué au taux de salaire horaire. Les deux schémas existent dans la littérature mais nous ne soulignerons pas la distinction lorsqu'elle n'a pas d'influence sur le contenu de notre argumentaire.

2. S'agissant de la terminologie, nous utiliserons parfois le terme d'honoraire de résultat pour qualifier les systèmes de rémunération à la performance que constituent l'honoraire contingent et l'honoraire conditionnel.

3. Le lecteur peut notamment se référer à Deffains et Langlais (2009) pour une présentation exhaustive du domaine qu'est l'économie du droit. 
suivant les contraintes auxquelles il est confronté. Dans cette optique, et suivant un prisme normatif, notre problématique centrale est alors de déterminer si l'honoraire de résultat est source d'effets positifs qui justifieraient son extension dans d'autres juridictions, telles que la France, l'Allemagne, le Portugal ou l'Italie, cette question revêtant une dimension sociétale forte. ${ }^{4}$

A cet égard, il semblerait que ce schéma de rémunération, dans sa déclinaison conditionnelle ou contingente, présenterait plusieurs avantages, qui pourraient justifier sa généralisation. Premièrement, l'honoraire de résultat améliorerait l'efficience de la relation avocat-client en limitant le phénomène d'aléa moral propre à cette relation : comparativement à un système de rémunération horaire, l'avocat serait incité à fournir un effort plus important pour défendre le cas juridique considéré, dans la mesure où il n'est rémunéré qu'en cas de victoire. Nous retrouvons ici un argument standard en faveur des mécanismes de rémunération variable (ou à la performance) au sein de la relation de travail, tel qu'il a été mis en lumière par la théorie des contrats (Laffont et Martimort, 2002). Deuxièmement, ces mécanismes favoriseraient l'accès à la justice des clients soumis à une contrainte budgétaire ne leur permettant pas de faire appel à un avocat rémunéré de manière horaire. En effet, lorsque la rémunération est liée à l'issue de la procédure, le client est amené à rétribuer son avocat uniquement s'il gagne le procès ou obtient une transaction (c'està-dire s'il perçoit des dommages-intérêts), la rémunération de son avocat venant en déduction de cette compensation. L'honoraire de résultat pourrait ainsi avoir vocation à se substituer au mécanisme public d'aide juridictionnelle impliquant la prise en charge des frais de justice par l'Etat. Troisièmement, la rémunération liée au résultat constituerait un mécanisme de transfert du risque - encouru lors du procès - du client vers son avocat. Ce transfert peut apparaître comme étant Pareto-améliorant dans la mesure où l'avocat peut a priori disposer d'un "portefeuille" de clients lui permettant d'adopter une politique de diversification. Enfin, l'honoraire de résultat pourrait constituer un mécanisme efficace de sélection des affaires selon leur probabilité de déboucher sur une issue favorable lors du procès. Un tel processus de sélection aurait l'avantage de permettre un certain désencombrement des tribunaux et de diminuer ainsi le coût social de fonctionnement du système judiciaire de résolution des litiges. L'ensemble de ces arguments peut rendre potentiellement intéressant la mise en oeuvre de schémas alternatifs de rémunération des avocats ayant possiblement des effets bénéfiques. Ces propos doivent néanmoins êtres nuancés, et ce pour trois raisons principales. Tout d'abord, comme cela est détaillé dans l'article, certaines conditions restrictives doivent parfois être remplies pour que les effets attendus apparaissent. Par ailleurs, la mise en oeuvre de l'honoraire de résultat peut avoir certains effets pervers. A titre d'exemple, il est possible que la rémunération contingente génère un conflit d'intérêts entre l'avocat et son client, le premier souhaitant obtenir une transaction amiable (afin de percevoir un gain immédiat sans assumer le coût d'un procès éventuel), potentiellement au détriment de son client. Enfin, il nous faut souligner que la très grande majorité des analyses existantes sur cette question sont d'ordre théorique, rendant délicat le fait d'en induire des recommandations de politique publique de manière trop affirmée. Ce biais présent dans la littérature s'explique certainement par la difficulté d'accéder à des données en la matière, de par le degré de confidentialité associé à la

4. Le recours à l'honoraire de résultat est possible en France ou en Allemagne mais ne constitue pas la norme en matière de rémunération des avocats (Baumann et Friehe, 2012). 
question de la résolution des litiges et de la rémunération des avocats, ce qui ouvre potentiellement un horizon de recherche via le recours à l'économie expérimentale.

Le reste de l'article est constitué de trois sections. La section 2 porte sur l'analyse de l'effet de l'honoraire de résultat sur le comportement des parties prenantes lors de la phase de résolution du litige, qui est constituée de la tentative de règlement amiable et du procès éventuel. La section 3 s'intéresse à l'étude des effets, plus indirects, sur l'émergence d'un litige (via l'impact sur la probabilité de survenance d'un accident) et sur l'incitation des justiciables à intenter une action en justice. La section 4 conclut.

\section{ModAlitéS DE RÉMUnÉRATION ET RÉSOLUTION DES LITIGES}

Dans cette section, nous aborderons successivement les deux étapes relatives au règlement d'un différend, à savoir la phase durant laquelle les parties tentent de se concilier dans la perspective d'une transaction amiable (section 2.1), puis la phase du procès à l'issue duquel une décision est prise par le tribunal, notamment sur la base de l'effort réalisé par l'avocat (section 2.2).

\subsection{Tentative de conciliation et règlement amiable}

Une littérature relativement vaste a pour objectif de déterminer si l'honoraire contingent est plus favorable au règlement amiable des litiges que d'autres mécanismes de rémunération. A cet égard, l'évaluation relative d'un type d'honoraire peut se fonder sur plusieurs critères, parfois contradictoires. En effet, si un avocat est rendu moins exigeant dans la négociation par un certain type d'honoraire, cela peut induire un taux d'arrangement plus élevé, ce qui peut apparaître souhaitable dans une perspective de minimisation des coûts de justice. Néanmoins, une telle configuration peut nuire au client, dans la mesure où il sera ainsi amené à percevoir un gain plus faible. Sur ce dernier point, la lecture de la littérature ne permet pas de parvenir à un consensus : certains articles démontrent un effet négatif ou ambigu de l'honoraire contingent sur les montants obtenus par les demandeurs au sein de la négociation, tandis que d'autres aboutissent à un effet positif. Certains auteurs proposent par ailleurs une analyse plus normative, en essayant d'identifier quelle forme d'honoraire contingent serait la plus à même de maximiser le bien-être des clients, en distinguant notamment honoraire contingent unitaire et binaire. ${ }^{5}$

2.1.1. Effet négatif de l'honoraire contingent sur les gains des clients. Plusieurs auteurs estiment que l'honoraire contingent devrait conduire à un taux de résolution amiable des litiges plus élevé que d'autres types d'honoraires, et ce au détriment de l'intérêt des clients, à savoir les demandeurs. Le principal argument est le suivant : sous un honoraire contingent, les avocats préfèrent négocier plutôt que d'aller au procès dans la mesure où ils perçoivent ainsi le pourcentage prévu sur le montant de l'arrangement sans avoir à fournir les efforts nécessaires à la poursuite de l'affaire devant les tribunaux (Gravelle et Waterson, 1993). Dans cette perspective, l'honoraire

5. L'honoraire contingent est dit unitaire si le pourcentage perçu par l'avocat est indépendant de l'issue du litige; a contrario, si l'honoraire est binaire, le pourcentage perçu par l'avocat en cas d'arrangement est différent de celui obtenu en cas de victoire au procès. 
contingent devrait conduire à des taux d'arrangement plus élevés et à des montants négociés plus faibles que ceux satisfaisant l'intérêt des clients (et obtenus via un honoraire horaire ou au temps passé). ${ }^{6}$

Le mécanisme à l'oeuvre attestant de l'impact négatif de l'honoraire contingent sur le paiement du client est exposé de façon simple par Spier (2007). Selon cet auteur, la résolution amiable d'un litige est conditionnée par l'existence d'un intervalle de négociation défini par la différence entre les valeurs de réserve des parties, dont la borne inférieure correspond au montant minimal accepté par le demandeur pour renoncer au procès et la borne supérieure à l'offre maximale que le défendeur serait prêt à payer pour éviter le procès. ${ }^{7}$ Dans une situation où le demandeur délègue sa décision de conclure un accord à son avocat, la borne inférieure de l'intervalle de négociation ne correspond plus à la demande minimale du demandeur mais à celle de son avocat. Or, il n'y a aucune raison de penser que ces deux valeurs sont identiques. Comme le montre Spier, ces valeurs dépendent notamment de la part des frais de procès que l'avocat assume (notée $\alpha$ ) et du pourcentage de rémunération qu'il perçoit en cas d'arrangement ou de victoire au procès (noté $\theta$, en supposant un honoraire unitaire). Ainsi, en notant $x$ le montant du jugement et $c_{P}$ le montant des frais de procès, l'offre amiable qui rendrait l'avocat indifférent entre procès et arrangement, notée $S_{A}$, est telle que $\theta x-\alpha c_{P}=\theta S_{A} \Leftrightarrow S_{A}=x-(\alpha / \theta) c_{P}$. Symétriquement, la valeur de l'offre qui rendrait le client indifférent entre procès et arrangement, notée $S_{P}$, est telle que $(1-\theta) x-(1-\alpha) c_{P}=(1-\theta) S_{P} \Leftrightarrow S_{P}=x-[(1-\alpha) /(1-\theta)] c_{P}$. Si $\alpha=\theta$, les intérêts $\mathrm{du}$ client et de son avocat sont parfaitement alignés puisqu'ils ont le même seuil d'acceptation : $S_{A}=S_{P}=S=x-c_{P}$. En revanche, dès lors que la part des frais de procès supportés par l'avocat excède son pourcentage de rémunération $(\alpha>\theta)$, ce qui correspond au cas standard, alors l'offre minimale que le client serait prêt à accepter excède celle de l'avocat, situation traduisant l'existence d'un conflit d'intérêts : $S_{A}<S<S_{P}$. En conséquence, la question de l'identité du détenteur du pouvoir décisionnaire est cruciale. L'honoraire contingent conduirait ainsi à un taux d'arrangement plus élevé et à un montant négocié plus faible lorsque l'avocat détient le pouvoir de décision, et inversement. ${ }^{8}$

Dans une perspective similaire, Miller (1987) compare le montant de l'arrangement sous l'honoraire contingent et l'honoraire au temps passé, en fonction du détenteur du pouvoir décisionnaire. Il montre, à partir d'un modèle simple de négociation inspiré de ceux de Landes (1971) et Posner (1973), que l'honoraire contingent ne conduit pas à un arrangement optimal, sauf si le demandeur dispose du pouvoir de décision. Dans ce cas en effet, la somme des gains du demandeur et de son avocat en acceptant une offre amiable est supérieure à la somme obtenue en cas de procès (de par l'économie des frais de procès réalisée), cette issue étant également préférable pour le défendeur et son avocat qui échappent aussi aux coûts de justice. A contrario, suivant les arguments précédents,

6. Il faut noter que l'honoraire perçu par l'avocat peut également être forfaitaire, et non horaire, mais nous mentionnerons rarement la distinction dans la mesure où les incitations véhiculées par ces deux modes de rémunération sont très souvent similaires.

7. Par "offre", nous entendons le montant proposé par le défendeur ou exigé par le demandeur durant la tentative de règlement amiable du différend.

8. Détenir le pouvoir de décision implique le fait de choisir le montant de l'offre à formuler/accepter et le fait de décider d'aller au procès ou non. 
l'avocat rémunéré de manière contingente subit les coûts de procès et sera donc incité à conclure un arrangement que son client aurait refusé s'il disposait du pouvoir décisionnaire. De la même manière, le client possédant le pouvoir de décision sous un honoraire horaire conclurait un arrangement nuisible à son avocat, ce dernier ayant intérêt à emmener l'affaire le plus loin possible.

Thomason (1991) propose de tester empiriquement les résultats de Miller (1987), à partir d'un échantillon constitué d'affaires impliquant des ouvriers new-yorkais. Il enrichit le modèle de Miller en intégrant le fait que les parties font des erreurs d'estimation sur le jugement attendu (traduisant des biais d'optimisme ou de pessimisme) et peuvent être averses au risque, notamment les demandeurs, ce qui les conduit à accepter des offres inférieures au paiement espéré au procès. ${ }^{9}$ Sur le plan théorique, il conclut que les demandeurs qui ont délégué leur pouvoir décisionnaire à leur avocat négocient plus fréquemment que les autres et acceptent des arrangements plus faibles. Il confirme empiriquement ces résultats, après avoir pris en compte les biais de sélection liés au fait que les individus pessimistes (resp., averses au risque) négocient plus souvent que les individus optimistes (resp., neutres ou risquophiles).

Enfin, Polinsky et Rubinfeld (2002) élaborent également un argumentaire selon lequel l'honoraire contingent nuirait aux clients. Ils utilisent un modèle dans lequel le demandeur, non informé sur les coûts du défendeur, lui fait une offre à prendre ou à laisser. Ils comparent l'honoraire contingent (l'avocat disposant dans ce cas du pouvoir décisionnaire) à une situation de référence dans laquelle le client choisirait lui-même l'offre à faire au défendeur et rémunèrerait son avocat au temps passé. Ils montrent que sous un honoraire contingent, l'avocat, qui supporte l'intégralité du coût du temps consacré à l'affaire mais ne perçoit qu'une partie du bénéfice qui en résulte, consacre au procès un temps de travail plus faible que si le client avait le pouvoir décisionnaire. Ainsi, le coût espéré du défendeur en cas de procès est plus faible, ce qui réduit son incitation à accepter une offre donnée. En conséquence, un premier effet est que le bénéfice marginal pour l'avocat d'augmenter l'offre est plus faible. Cependant, si le défendeur rejette l'offre, le coût pour l'avocat d'aller au procès est également plus faible puisque le nombre d'heures est réduit par rapport à la situation de référence; ce second effet (baisse du coût) va à l'encontre du premier (baisse du bénéfice). Globalement, Polinsky et Rubinfeld (2002) montrent que le second effet l'emporte sur le premier, ce qui implique une offre plus faible de la part de l'avocat sous honoraire contingent (traduisant une incitation insuffisante à obtenir un arrangement).

2.1.2. Effet positif de l'honoraire contingent sur les gains des clients. Contrairement aux travaux précédents, d'autres auteurs démontrent que l'honoraire contingent peut jouer en faveur du client lors de la négociation d'un accord amiable, y compris lorsque l'avocat dispose du pouvoir décisionnaire. Ainsi, Bebchuk et Guzman (1996) analysent la façon dont le type d'honoraire, contingent ou au temps passé, affecte le montant de l'arrangement. Ils soutiennent que sous un honoraire au temps passé, le client préfèrera négocier plutôt que d'aller au procès, pour réduire le nombre d'heures travaillées de l'avocat (et ainsi la rémunération à lui verser). Sous un honoraire contingent, le fait que le client ne supporte pas de coûts en cas de procès implique que sa valeur

9. Un protagoniste, demandeur ou défendeur, est dit optimiste (resp. pessimiste) si son estimation de la probabilité qu'il a de gagner le procès est supérieure (resp. inférieure) à la probabilité objective. 
de réserve est plus élevée en honoraire contingent qu'en honoraire au temps passé. Cela lui confère un avantage stratégique, dans la mesure où le défendeur sera contraint de lui proposer une offre de règlement amiable plus élevée. Si la rémunération de l'avocat est intégrée dans le modèle, alors le raisonnement précédent reste valide si le contrat entre le client et son avocat précise un honoraire contingent égal à la rémunération que l'avocat aurait perçue jusqu'à la phase de négociation en cas d'honoraire au temps passé. De ce point de vue, l'honoraire contingent permet au demandeur d'obtenir des arrangements plus favorables que l'honoraire au temps passé, dans la mesure où ils le laissent indifférent entre le procès et la résolution amiable du litige, puisque le transfert de coûts du client vers l'avocat renforce le pouvoir de négociation du client supposé détenir le pouvoir décisionnaire. Rickman (1999) prolonge l'analyse de Bebchuk et Guzman (1996) par un modèle théorique avec asymétrie d'information entre le défendeur et l'avocat du demandeur sur le mérite de l'affaire. ${ }^{10}$ Le cadre retenu est dynamique, le défendeur pouvant faire une deuxième offre à prendre ou à laisser au demandeur si ce dernier a refusé la première. Il part du constat que dans les modèles qui démontrent l'impact négatif de l'honoraire contingent (Miller, 1987; Gravelle et Waterson, 1993), les coûts supportés par l'avocat en cas de procès affaiblissent sa position de négociation et le conduisent à négocier des accords que son client n'aurait pas acceptés. ${ }^{11}$ Par leur caractère statique, ces modèles n'intègrent pas l'influence combinée de l'honoraire contingent et des coûts de procès, ni la possibilité pour l'avocat d'adopter un comportement stratégique construit sur plusieurs périodes. Plus précisément, l'avocat qui adopte une stratégie agressive dans la négociation, en refusant une offre, oblige ensuite l'adversaire à faire une proposition plus élevée parce qu'il signale, par son refus, qu'il est prêt à prendre le risque de supporter des coûts de négociation supplémentaires, voire d'aller au procès, pour obtenir davantage. Autrement dit, le refus permet de signaler à l'adversaire que l'affaire est solide. Cette stratégie lui permet d'obtenir des montants d'arrangements plus élevés. Il n'est cependant pas certain que le demandeur bénéficie de ce montant d'arrangement plus élevé dans la mesure où l'avocat est incité à compenser les coûts de négociation élevés de deux façons : d'une part, par une hausse de l'offre acceptée (qualifiée d'effet coût) et d'autre part, par l'honoraire contingent requis pour couvrir son coût d'opportunité, ce qui contrebalance les bénéfices que le client pourrait retirer de cette stratégie.

Casagrande et Spallone (2007) proposent un cadre d'analyse différent : ils élaborent un modèle à quatre joueurs (les clients et leurs avocats respectifs), et recherchent les conditions sous lesquelles un équilibre de Nash existe, en tenant compte d'un taux d'escompte qui s'applique si l'affaire n'est résolue qu'avec un procès. Comme les auteurs précédents, ils comparent les incitations à négocier sous un honoraire au temps passé et sous un honoraire contingent, mais en se fondant sur l'ampleur de l'intervalle de négociation obtenu à l'équilibre, selon le mode de rémunération de l'avocat. ${ }^{12}$ Plus cet intervalle est grand, plus les chances de parvenir à un accord sont supposées élevées, chacun ayant intérêt à accepter les offres qui y sont incluses. Les auteurs montrent que l'hono-

10. Il s'agit ici du mérite juridique, capturé par exemple par la probabilité de victoire au procès (voir la section 3.2 pour une définition plus précise).

11. Cet impact est négatif chez Miller (1987) seulement dans le cas où l'avocat a le pouvoir de décision.

12. L'intervalle de négociation représente l'ensemble des propositions d'arrangement acceptables par l'ensemble des parties, c'est-à-dire le demandeur, le défendeur et leurs avocats respectifs. Dès lors qu'une offre se situe dans cet intervalle, le conflit est considéré comme étant résolu à l'amiable. 
raire horaire favoriserait le règlement amiable des litiges relativement à l'honoraire contingent en impliquant un intervalle de négociation plus grand, mais seulement sous certaines conditions. En particulier, le taux de rémunération de l'avocat en cas d'arrangement, supposé plus élevé que le taux (actualisé) en cas de procès, ne doit pas être trop élevé par rapport à ce dernier pour que le résultat tienne; en outre, le résultat principal tient également au fait que les anticipations sur les résultats du procès soient divergentes entre le couple demandeur-avocat d'un côté, et le couple défendeur/son avocat de l'autre.

2.1.3. Honoraire contingent unitaire versus binaire. Face à cette absence de consensus quant à l'impact de l'honoraire contingent sur la négociation, plusieurs auteurs ont adopté une approche de portée plus normative, portant sur la forme de l'honoraire contingent permettant de rendre la négociation plus profitable du point de vue du demandeur.

La première analyse relative au schéma d'honoraire contingent permettant de maximiser à la fois le montant obtenu par le couple demandeur/son avocat et la part obtenue par le client luimême est proposée par Hay (1997). Celui-ci considère deux schémas de rémunération possibles : un schéma "unitaire", le plus couramment utilisé selon l'auteur, et un schéma "binaire" (bifurcated), dans lequel le taux de rémunération de l'avocat est supposé plus faible si le conflit est résolu à l'amiable que s'il est tranché par le juge. Il montre que le schéma de rémunération le plus efficace eu égard aux critères précédemment cités serait un honoraire binaire dans lequel l'avocat obtiendrait un large pourcentage du gain en cas de procès et un faible pourcentage en cas d'arrangement. En effet, ce schéma présenterait un double avantage : un pourcentage élevé au procès permettrait de générer ex-ante un montant d'arrangement élevé, tandis qu'un pourcentage faible en cas d'arrangement éviterait au client de payer son avocat pour un effort (durant le procès) qu'il n'aura pas à fournir, évitant ainsi de lui verser une rente. Cependant, ces résultats dépendent, d'une part, de l'allocation du pouvoir décisionnaire lors de l'arrangement entre le demandeur et son avocat, et d'autre part, du pouvoir de négociation relatif entre le demandeur et le défendeur. ${ }^{13}$ En effet, les avantages d'un honoraire binaire sont les plus élevés soit lorsque l'avocat détient le contrôle dans la décision d'arrangement, soit lorsque c'est le client qui le détient mais que son pouvoir dans la négociation (relativement à celui du défendeur) est faible.

Choi (2003) recherche également la forme d'honoraire contingent la plus adéquate pour le demandeur en partant du double avantage mis en relief par Hay (1997) concernant un taux de rémunération de l'avocat élevé en cas de procès et faible en cas d'arrangement. Cependant, Choi étend la précédente analyse en intégrant la problématique de la rente de l'avocat et se demande en particulier si le client a nécessairement intérêt à éliminer cette rente ( via le versement d'un pourcentage faible en cas d'arrangement). Si le demandeur dispose du pouvoir de décision, le fait de verser un pourcentage faible lui permet alors de minimiser les coûts supportés mais réduit en même temps son pouvoir de négociation vis-à-vis du défendeur. En effet, la baisse des coûts évoquée amène le demandeur à être prêt à accepter une offre plus faible, ce que le défendeur est à même d'anticiper et d'exploiter. A l'inverse, lorsque le demandeur délègue la négociation

13. Le montant négocié lors de l'arrangement entre les parties se situe entre leurs valeurs de réserve respectives, la valeur de réserve de chacune étant pondérée par un paramètre représentant son pouvoir de négociation. 
à son avocat, réduire la fraction qui lui est versée lors de l'arrangement renforce sa position de négociation vis-à-vis du défendeur. Le montant négocié dépend à présent de la valeur de réserve de l'avocat qui est plus élevée lorsque le pourcentage perçu sur l'arrangement amiable est plus faible. L'offre du défendeur doit donc être plus grande pour être acceptée par l'avocat, impliquant que le client tire pleinement avantage de l'arrangement. Parallèlement, l'avocat obtient également une rente non négligeable, puisque son gain issu de l'arrangement est plus élevé que celui qu'il obtiendrait au procès ( $\mathrm{du}$ fait du caractère binaire de la rémunération). Contrairement à Hay (1997), Choi (2003) démontre ainsi que l'honoraire contingent permet toujours d'éliminer la rente de l'avocat ou la rente du défendeur, et ce quels que soient le pouvoir de négociation du client et l'allocation du pouvoir décisionnaire

Farmer et Pecorino (2005) prolongent l'analyse en développant un modèle de signal, fondé sur l'approche de Reinganum et Wilde (1986), dans lequel le demandeur fait une offre d'arrangement amiable au défendeur en ayant une information privée quant à la valeur du jugement, notée $x$, obtenu en cas de procès (son avocat et le défendeur ne connaissant que la distribution des "types"). En honoraire fixe, le demandeur encourt des frais de procès notés $c_{P}$ tandis qu'en honoraire contingent, il rémunère son avocat $\theta x$ si le procès est gagné (ce qui survient avec une probabilité $p$ ) et rien si le procès est perdu (avec une probabilité $1-p$ ). Ainsi, en honoraire contingent, un demandeur de type faible $\left(x<c_{P} / p \theta\right)$ est confronté à un coût de procès espéré plus faible qu'un plaignant du même type en honoraire fixe, ce qui réduit le coût de voir une offre donnée rejetée par le défendeur. En conséquence, le taux de rejet d'équilibre de l'offre doit initialement croître à un taux plus élevé que sous un honoraire fixe afin d'induire des offre pleinement révélatrices. Cela explique qu'un contrat à honoraire binaire induise davantage de rejets d'offres, et donc davantage de procès, parmi les demandeurs de type faible. Pour des raisons symétriques, le taux d'arrangement serait plus élevé sous un honoraire binaire que sous un honoraire fixe, pour un type de demandeur suffisamment élevé. En poursuivant leur analyse, Farmer et Pecorino montrent que l'honoraire contingent modifie la sélection des affaires puisqu'il emmène jusqu'au procès davantage de cas à faible enjeu qu'un honoraire fixe. En outre, sous un honoraire unitaire, le demandeur rémunère son avocat à un pourcentage identique quelle que soit l'issue du litige, ce qui réduit le coût relatif d'aller au procès. En conséquence, la probabilité d'arrangement est plus faible en honoraire unitaire qu'en honoraire fixe ou binaire. ${ }^{14}$

Leshem (2009) étend l'analyse précédente en supposant que l'avocat peut disposer du pouvoir décisionnaire, à savoir la possibilité de formuler l'offre à prendre ou à laisser destinée au défendeur. Selon l'auteur, cette délégation de pouvoir bénéficie au demandeur, en lui permettant d'obtenir une probabilité d'arrangement et un paiement d'équilibre plus élevés. L'intuition est la suivante : l'honoraire contingent rendant l'avocat plus réticent à aller au procès que son client, le défendeur est moins capable d'induire la révélation du vrai type du demandeur en menaçant de mettre fin aux négociations que sous un honoraire fixe. Ainsi, la réticence de l'avocat à aller au procès lui permettrait de mieux signaler le type de son client à travers l'offre d'arrangement qu'il proposerait au défendeur. En conséquence, la probabilité de conclure un arrangement est supérieure à celle

14. Notons que l'extension du modèle au cas où un défendeur informé ferait l'offre génère des résultats qualitativement similaires : la probabilité d'arrangement est la plus faible sous un honoraire unitaire. 
observée sous un honoraire au temps passé parce que le défendeur est capable, à travers l'offre de l'avocat, d'anticiper correctement le résultat du procès. Cet article contraste ainsi avec ceux de Bebchuk et Guzman (1996) et Choi (2003) qui montrent que les demandeurs devraient conserver le pouvoir décisionnaire sous honoraire contingent afin d'améliorer leur pouvoir de négociation vis-à-vis du défendeur. Les différences en termes de résultats s'expliquent par le fait que Leshem suppose que le demandeur possède tout le pouvoir de négociation, et se focalise ainsi sur le rôle de l'information asymétrique dans les négociations.

\subsection{Effort de l'avocat et problème d'aléa moral}

De manière générale, il existe à ce jour un certain consensus dans la littérature théorique sur le fait que l'honoraire de résultat, qu'il soit contingent ou conditionnel, améliore l'efficience de la relation avocat-client en limitant le phénomène d'aléa moral inhérent à cette relation : comparativement à un système de rémunération fixe ou horaire, l'avocat serait incité à fournir un effort plus important pour défendre le cas juridique en question dans la mesure où il n'est rétribué qu'en cas d'arrangement ou de victoire au procès. Plusieurs articles démontrent cette propriété des mécanismes de rémunération à la performance dans un contexte d'effort inobservable (voir notamment Halpern et Turnbull, 1983; Danzon, 1983; Gravelle et Waterson, 1993; Hay, 1996; Polinsky et Rubinfeld, 2003). Cette propriété est par ailleurs vérifiée empiriquement par McKee, Santore, et Shelton (2007) qui constitue, à notre connaissance, la seule étude empirique sur cette question. ${ }^{15}$ Etant donné la difficulté de mesurer le niveau d'effort sur la base de données de terrain, les auteurs mobilisent la méthode expérimentale et montrent que l'effort des avocats est effectivement significativement croissant avec le taux de rémunération perçu dans le cadre d'un honoraire contingent. Sur cette base, il apparaît que les avocats sont susceptibles d'obtenir une rente dans la mesure où la rémunération moyenne obtenue dans l'expérience, qui reproduit l'existence d'un marché concurrentiel entre avocats, est plus élevée que la rémunération concurrentielle théorique : conscients des vertus incitatives d'un honoraire contingent suffisamment généreux, les clients n'ont en effet pas intérêt à mettre en oeuvre des pourcentages de rémunération contingente saturant la contrainte de participation de leurs avocats.

Dans une perspective comparative, Emons et Garoupa (2006) analysent les vertus incitatives de l'honoraire contingent et de l'honoraire conditionnel dans le cadre d'une relation principal-agent au sein de laquelle l'avocat choisit son niveau d'effort après avoir pris connaissance de l'enjeu du litige, à savoir le montant des dommages-intérêts attendus. ${ }^{16}$ Les auteurs supposent que l'avocat dispose d'une information privée non seulement sur son niveau d'effort, suivant en cela l'hypothèse d'aléa moral, mais également sur l'enjeu du litige, ce qui peut se justifier par son degré d'expertise. Dans ce cadre, il est démontré que l'honoraire de résultat, qu'il soit contingent ou conditionnel, est incitatif à l'effort comparativement à l'honoraire fixe. Cependant, la rémunération condition-

15. Suivant un argument similaire, Chappe et Gabuthy (2013) montrent que la mise en oeuvre de l'honoraire contingent est également souhaitable lorsque le litige implique le recours à un arbitre, et non à un juge, l'arbitre étant une personne privée choisie par les parties en conflit pour obtenir une décision dans le cadre d'une procédure extrajudiciaire.

16. Dans ce type de modélisation, le client est le principal (non informé), tandis que l'avocat est l'agent (informé), et l'asymétrie d'information porte sur le niveau d'effort choisi par ce dernier. 
nelle apparaît dominée par la rémunération contingente du fait de la structure informationnelle de l'interaction. En effet, l'honoraire contingent permet à l'avocat d'exploiter son avantage informationnel, puisqu'il perçoit une part des dommages-intérêts dont il est le seul à connaitre le montant. L'avocat est ainsi incité à ajuster son niveau d'effort à ce montant, ce qui n'est pas le cas sous honoraire conditionnel dans la mesure où sa rémunération est indépendante de la valeur des dommages-intérêts (impliquant simplement le versement d'un bonus forfaitaire en cas de victoire au procès). La rémunération contingente maximiserait ainsi le surplus social et donc l'efficience de la relation entre l'avocat et son client. Cette supériorité de l'honoraire contingent est également mise en avant par Hyde (2006) suivant un argument différent : dans le cadre d'une rémunération conditionnelle impliquant une rémunération horaire à laquelle s'ajoute un bonus s'appliquant sur chaque heure effectuée, l'auteur avance l'idée que ce schéma de rémunération pourrait générer un sur-investissement (sous-optimal) de l'avocat en termes d'effort. ${ }^{17}$ Il faut néanmoins noter que ce comportement, qualifié d'over-servicing ou de cost-padding, peut être régulé par le client dont le niveau d'expertise n'est pas nécessairement nul.

Dans le cadre d'une analyse normative, Wang (2008) démontre même que la rémunération contingente à l'américaine, impliquant un taux de rémunération compris entre 0 et 1 exclus, permet d'atteindre l'optimum de premier rang. Le phénomène d'aléa moral serait donc totalement éliminé par la mise en place de ce mécanisme de rémunération. Il faut néanmoins noter que ce résultat est obtenu sous des hypothèses relativement restrictives, tel qu'un coût marginal de l'effort supposé constant, lui conférant un degré de généralité potentiellement limité. A cet égard, il semble pertinent de considérer qu'un honoraire contingent optimal consisterait plutôt à transférer l'affaire du client vers l'avocat, impliquant la mise en oeuvre d'un pourcentage de rémunération de $100 \%$ et d'un versement forfaitaire de l'avocat au client. Suivant un argument évoqué dans la section 2.1.1, l'incitation à l'effort de l'avocat serait ainsi maximale dans la mesure où il ne serait pas contraint de partager les bénéfices résiduels du recours en justice, à savoir le montant des dommagesintérêts obtenus. Même si un tel mécanisme était autorisé (ce qui n'est pas le cas), Spier (2007) souligne néanmoins plusieurs arguments en sa défaveur : le volume d'actions en justice pourrait être excessif, les avocats ayant potentiellement une incitation plus forte à obtenir le versement de dommages-intérêts ; par ailleurs, le succès d'une action en justice repose très certainement sur une forme de coopération mutuelle au sein du binôme avocat-client, coopération a priori optimisée par la mise en place d'un taux de rémunération contingente compris entre 0 et 1 (permettant de partager les bénéfices de l'action en justice).

Au-delà de ce consensus, l'ensemble de ces arguments va néanmoins potentiellement à l'encontre d'un fait stylisé, à savoir la tendance des grands cabinets d'avocats américains à préférer que leurs prestations soient rémunérées sous la forme d'une indemnisation horaire plutôt que contingente. Hadfield (2000) met en avant la segmentation du marché des avocats aux EtatsUnis qui se caractérise par la coexistence de deux secteurs. Le premier (personal client segment) concerne les avocats exerçant de manière isolée, dont la clientèle est principalement constituée

17. Comme nous le mentionnons en introduction, l'honoraire conditionnel, tel qu'il est appréhendé dans la littérature, peut prendre la forme d'un surcroît de rémunération horaire ou d'un bonus forfaitaire indépendant du nombre d'heures travaillées. 
de particuliers et dont les affaires relèvent d'une forme de routine (divorces, expulsions locatives, successions, ...). Le second (corporate segment) est constitué de grands cabinets dont les affaires sont prestigieuses et qui recrutent des avocats influents dans la profession et diplômés d'universités renommées. Dans le cadre d'actions en responsabilité civile, les observations empiriques montrent que les avocats de ce second secteur sont principalement rémunérés à l'heure, tandis que leurs confrères du premier segment perçoivent des indemnisations contingentes à leurs résultats. S'intéressant au second segment, Garoupa et Gomez-Pomar (2007) avancent une explication possible à ce phénomène : dans le cadre d'un modèle d'aléa moral avec multi-principaux, ils montrent que l'introduction d'un mécanisme de rémunération contingente permet certes d'aligner les intérêts de l'avocat avec ceux du client mais nuit potentiellement à l'efficience de la relation entre cet avocat et le cabinet auquel il appartient. Sous ce régime d'indemnisation, l'avocat est en effet incité à exercer un niveau d'effort excessif, coûteux pour le cabinet dans la mesure où ce travail mobilise des ressources communes (secrétariat, assistance juridique, matériel informatique, ...). Il est alors possible d'identifier une configuration dans laquelle un système de rémunération horaire est préférable à une rétribution contingente du point de vue du cabinet, ce dernier étant naturellement confronté à un arbitrage entre les effets bénéfiques de l'effort en termes de gains et le coût de cet effort en termes d'utilisation des ressources matérielles. Ce coût n'est pas pris en compte par l'avocat et constitue une pure externalité de ce point de vue.

Par ailleurs, dans une perspective différente, At et Gabuthy (2015) montrent que l'optimalité de l'honoraire contingent, tel qu'il existe, peut être altérée dans un contexte de procès séquentiel (sequential litigation). Suivant cette configuration, le tribunal détermine tout d'abord le degré de responsabilité du défendeur (phase 1), puis fixe ensuite le montant des dommages-intérêts à verser (phase 2), impliquant une relation d'agence dynamique entre l'avocat et son client. ${ }^{18}$ Les auteurs montrent que la nature séquentielle de l'interaction implique une structure contractuelle particulière : à l'optimum de second rang, l'avocat doit obtenir une rémunération nulle à l'issue de la phase 1 dans la mesure où la perspective de paiement prévu en phase 2 , sous forme d'un honoraire contingent, suffit à générer les incitations adéquates. A notre connaissance, cette structure contractuelle ne correspond à aucun dispositif existant actuellement.

Cette section a globalement permis de mettre en lumière la manière dont la littérature appréhende l'impact de l'honoraire de résultat sur les comportements durant les différentes étapes de résolution d'un litige en responsabilité civile (comportements de négociation lors de la phase de conciliation et incitations à l'effort lors du procès). Il s'avère que ce mode de rémunération peut également structurer les incitations en amont d'une action en justice, influençant ainsi l'émergence potentielle de ce recours.

\section{Modalités De RÉmunÉration et ÉMERGEnCE DU Litige}

Cette partie est consacrée à l'analyse de l'impact des modalités de rémunération des avocats sur l'émergence des actions en responsabilité civile, via les implications que ces modalités peuvent

18. A titre d'exemple, le procès opposant Polaroid et Kodak dans les années 80, à propos du brevet relatif à la photographie instantanée, s'est déroulé selon cette séquentialité (Landes, 1993). 
avoir en matière d'accès au droit pour les justiciables (section 3.1), de sélection potentielle des affaires selon leurs mérites juridiques (section 3.2) et de survenance des accidents (section 3.3).

\subsection{Accès au droit}

Toute action en justice engendre des coûts qui peuvent constituer des obstacles à l'accès au droit. Plusieurs modalités de transfert de ces coûts vers un autre agent existent cependant : l'assurance de protection juridique, une aide financière totale ou partielle de l'Etat (pour les plus démunis), le financement par un tiers (pour certains types de conflits commerciaux), le crowdfunding (Perry, 2018), et enfin le système d'honoraire contingent impliquant l'existence d'un lien entre le mode de rémunération de l'avocat et l'accès au droit. Il semble, en particulier, que l'interdiction des honoraires contingents dans certains pays ait été justifiée à l'origine par la volonté de décourager les actions en justice (voir Dover, 1986, pour l'Angleterre et Faure, Fernhout, et Philipsen, 2010, pour d'autres pays). Aux Etats-Unis, c'est au contraire la volonté de permettre aux plus démunis de faire valoir leurs droits qui justifierait leur légalité (Rubinfeld et Scotchmer, 1998). Plus précisément, l'honoraire contingent (de même que l'honoraire conditionnel), améliorerait l'accès à la justice, l'avocat du demandeur ne recevant aucune rémunération si l'affaire est perdue mais un pourcentage des dommages-intérêts (ou une rémunération augmentée d'un bonus) en cas de victoire de son client (Lambert et Chappe, 2014). Deux approches comparatives se dégagent de la littérature concernant la relation entre la rémunération contingente de l'avocat et l'accès au droit du demandeur dans une perspective de transfert de coûts : la première consiste à comparer les systèmes de transfert de coût de procès (honoraire contingent, assurance de protection juridique, aide juridictionnelle, financement par un tiers) tandis que la seconde porte sur l'impact relatif des différents types d'honoraires (contingent, conditionnel, forfaitaire, horaire). D'autres éléments d'analyse sont également avancés concernant le transfert de risque entre le demandeur et son avocat, l'aversion pour le risque pouvant constituer une barrière à l'accès au droit. Enfin, la question se pose également de l'impact de ces différents modes de financement sur l'accès au droit des défendeurs.

3.1.1. Honoraire contingent versus autres modes de financement. En Europe, l'accès au droit est considéré comme un droit fondamental de sorte que les Etats prennent en charge tout ou partie des frais de justice des plus démunis, d'autres modes de financement étant à la disposition des justiciables plus fortunés. ${ }^{19}$ Aux Etats-Unis, en revanche, l'accès au droit, notamment des plus pauvres, est principalement garanti par la sphère privée, via la rémunération contingente des avocats. Les systèmes d'aide juridictionnelle (legal aid) et d'honoraire contingent peuvent alors apparaître comme poursuivant le même objectif. En effet, comme l'expliquent Gravelle et Waterson (1993), la rémunération contingente facilite l'accès à la justice, en relâchant la contrainte budgétaire de ceux qui ne sont pas éligibles à l'aide juridictionnelle. Pour autant, nous pouvons

19. Voir l'article 47 de la Charte des droits fondamentaux de l'Union Européenne. S'agissant du rôle de la richesse, Bouquin (2004) défend la mise en oeuvre d'un accès à la justice à trois étages en France : l'aide juridictionnelle pour les plus pauvres, l'assurance de protection juridique pour la majorité des Français et le recours direct à l'avocat pour les riches. 
nous interroger quant à leur complète substituabilité. Ainsi, Posner (1996) compare les systèmes anglais et américain et soutient que l'aide juridictionnelle n'est pas un substitut parfait de l'honoraire contingent dans la mesure où elle ne profite pas aux classes moyennes dont l'accès au droit est mieux garanti par un système de rémunération contingente. Lambert et Chappe (2014) ont montré, quant à elles, que la probabilité de poursuites est plus élevée sous un honoraire de résultat. En effet, le demandeur financièrement contraint est toujours incité à poursuivre sous ces système de rémunérations parce qu'il n'avance pas les frais de justice, alors qu'il les avance dans un système d'aide juridictionnelle (forfaitaire ou proportionnelle). ${ }^{20}$

La question de l'accès au droit ne concerne pas uniquement la population des plus démunis. Le développement du financement des procès par des tiers a également généré des travaux comparatifs. Ainsi, Faure et Mot (2012) se sont interrogés sur les conséquences du développement $\mathrm{du}$ financement des procès par les tiers sur l'accès au droit, dans les pays de Common Law où les honoraires de résultats sont autorisés à titre exclusif (honoraire contingent aux Etats-Unis) ou complémentaire (honoraire conditionnel au Royaume-Uni). Ils avancent que ce mode de financement, perçu comme complémentaire à la rémunération au résultat des avocats, pourrait accroître l'accès au droit notamment en raison de sa plus grande capacité à soutenir des affaires très risquées. C'est notamment le cas pour les actions de groupe (class actions) de grande ampleur pour lesquelles la capacité financière des firmes d'avocats peut être insuffisante (pour pouvoir les porter) alors que celle des tiers financeurs est potentiellement plus élevée. Par ailleurs, selon Rubin (2011), les économies d'échelle que peuvent réaliser les avocats dans la sphère juridique sont inférieures à celles que peuvent réaliser ces tiers financeurs dans la mesure où ils peuvent investir dans plusieurs affaires d'enjeu et de risque élevés, affaires qui seront plaidées par des avocats de firmes différentes. En considérant plutôt ces deux modes de financement comme des substituts, Deffains et Desrieux (2015) montrent que le financement par les tiers n'accroît pas nécessairement l'accès à la justice par rapport à l'honoraire contingent. Plus précisément, si l'accès à la justice est contraint par une barrière de coûts dans le cas de l'honoraire au résultat (la part du gain attendu du litige pour l'avocat doit permettre de couvrir ses frais), il l'est par une barrière de rentabilité dans le cas du financement par un tiers. En effet, le tiers financeur s'engage uniquement si l'affaire est non seulement rentable une fois intégrés les coûts de transaction supplémentaires par rapport à l'honoraire contingent mais l'est surtout davantage que des placements alternatifs. En effet, selon les auteurs, le financement des procès par un tiers est susceptible d'induire des coûts de transaction supplémentaires par rapport à une relation bilatérale entre un avocat et son client, coûts liés notamment à la nécessité de conclure un contrat supplémentaire entre le client et le tiers financeur, aux négociations portant sur le partage du contrôle de l'affaire (notamment le pouvoir de décision), à l'évaluation des risques par le tiers, ou encore à la résolution des conflits éventuels entre les trois parties prenantes (tiers financeur, avocat et client).

3.1.2. Honoraire contingent versus autres types d'honoraires. D'une manière générale, l'honoraire de résultat (contingent ou conditionnel) faciliterait l'accès au droit en levant la contrainte

20. L'aide juridictionnelle proportionnelle implique qu'une proportion des dépenses du demandeur est prise en charge par l'Etat, tandis que l'aide forfaitaire renvoie à l'existence d'un montant fixe venant s'ajouter aux dépenses du demandeur. 
financière des demandeurs contrairement aux autres mécanismes de rémunération (forfaitaire ou horaire). L'un des avantages de l'honoraire contingent est en effet de permettre aux individus qui ne pourraient pas payer un avocat à l'heure de bénéficier de ses services grâce à une rémunération sur la base du jugement attendu qui ne devient effective qu'en cas d'accord ou de procès favorable au client (Miceli et Segerson, 1991; Gravelle et Waterson, 1993; Spier, 2007). De plus, aux Etats-Unis, les avocats sont plus souvent réticents à accepter une affaire, notamment en matière de préjudice corporel, sur la base d'une rémunération horaire s'ils n'ont pas la certitude que le demandeur dispose de suffisamment d'actifs pour garantir leur rémunération; ils seront en revanche plus enclins à s'engager sur la base d'un honoraire contingent. Suivant Dover (1986), l'accès au droit serait donc mieux garanti par un système contingent que par un système au temps passé, même s'il est généralement plus coûteux pour le client, parce qu'il intègre à la fois une prime de risque (liée au fait que l'avocat assume le risque de perdre) mais aussi un taux d'intérêt (lié au fait que le service est offert avant son paiement). ${ }^{21}$

L'exercice du droit d'accès à la justice dépend non seulement des coûts qui en résultent mais aussi du résultat escompté et donc de la confiance que le justiciable accorde à celui qu'il charge de défendre ses intérêts. Contrairement aux autres modes de rémunération et comme nous l'avons vu précédemment, l'honoraire de résultat aligne a priori les intérêts de l'avocat sur ceux du client puisque leurs gains respectifs sont directement liés, et résout ainsi le problème d'aléa moral qui peut se poser entre les deux parties. Selon Dana et Spier (1993), les individus ayant subi un préjudice pourraient ainsi ne pas faire confiance à leur avocat dans un monde sans honoraire de résultat, notamment quant à son évaluation des véritables mérites de l'affaire (puisque la rémunération horaire comme forfaitaire lui garantit d'être payé même si l'affaire est perdue). Cela pourrait les dissuader d'intenter une action en justice et plus généralement faire reposer leur décision d'intenter des poursuites sur leur propre anticipation ex-ante des gains et du coût total attendus des poursuites. ${ }^{22}$ En conséquence, si le demandeur est optimiste, l'honoraire contingent réduit le nombre de poursuites judiciaires par rapport à un honoraire forfaitaire ou au temps passé parce que l'avocat corrige le biais d'optimisme de son client. En revanche, si le demandeur est pessimiste, il renonce à intenter des poursuites, alors qu'un avocat payé au résultat l'aurait incité à aller en justice, l'affaire générant en réalité des gains attendus supérieurs aux coûts.

3.1.3. Honoraire contingent et transfert du risque. L'aversion pour le risque peut également jouer un rôle dans le lien entre rémunération et accès au droit. En effet, même sans avoir une estimation biaisée du jugement attendu, un demandeur qui éprouve de l'aversion pour le risque peut renoncer à faire valoir ses droits du fait de l'existence d'une incertitude inhérente à l'issue

21. Danzon (1983) parvient cependant au résultat inverse selon lequel la fréquence des poursuites peut être supérieure sous un honoraire au temps passé parce qu'un avocat neutre au risque rémunéré au résultat peut être incité à refuser des affaires qui seraient portées en justice par un client neutre au risque payant son avocat à l'heure. Sous les hypothèses posées par l'auteur, ce résultat est obtenu si le demandeur externalise davantage de coûts lorsqu'il paye son avocat à l'heure que lorsqu'il le paye au résultat.

22. Selon les auteurs, si l'honoraire de résultat était interdit, le demandeur ne pourrait pas se fier à son avocat pour une évaluation précise du fond de l'affaire et la décision de poursuivre serait en grande partie indépendante des caractéristiques juridiques individuelles de l'affaire. 
du procès. L'honoraire contingent, contrairement aux autres types d'honoraires, permet alors de transférer le risque du demandeur vers son avocat qui peut perdre la valeur des services rendus s'il échoue (Dover, 1986; Emons et Fluet, 2016). Ce dernier est supposé avoir moins d'aversion pour le risque que son client puisqu'il peut diversifier son portefeuille de clients et donc mieux gérer le risque, la mutualisation pouvant s'opérer puisque les risques individuels sont indépendants (excepté dans les actions de groupe) et les firmes regroupent un grand nombre d'affaires (Gravelle et Waterson, 1993; Faure, Fernhout, et Philipsen, 2010). En conséquence, comme le rappellent Zamir et Ritov (2010), l'honoraire contingent est à la fois un crédit que l'avocat fait à son client, puisque le client ne paye qu'après perception des dommages-intérêts, et une assurance, puisqu'il ne paye pas si l'affaire est perdue. ${ }^{23}$ L'honoraire contingent permettrait ainsi, relativement à d'autres types d'honoraires, un partage plus efficient des risques, avec pour conséquence de faciliter l'accès au droit des demandeurs qui ont de l'aversion pour le risque. Les auteurs confirment empiriquement ces résultats. A partir de la théorie des perspectives de Kahneman et Tversky, ils étudient expérimentalement les préférences des parties concernant les honoraires d'avocat. L'honoraire fixe ou au temps passé confronte le demandeur à une loterie mixte (comprenant un gain et une perte) dans laquelle il peut soit gagner une certaine somme d'argent (correspondant à ses dommages-intérêts desquels il doit déduire les honoraires), soit supporter une perte (correspondant aux honoraires qu'il doit verser à son avocat s'il perd le procès) par rapport à sa situation de référence. ${ }^{24}$ En revanche, l'honoraire contingent élimine tout risque de perte puisque le demandeur ne paye pas d'honoraire si l'affaire échoue. La loterie est donc composée de gains positifs ou nuls. Lorsque le choix de l'individu porte sur une alternative composée d'une loterie mixte et d'une loterie positive, la théorie des perspectives prédit qu'il préfèrera l'honoraire contingent. En effet, suivant cette approche, les individus raisonnent par rapport à leur point de référence et expriment de l'aversion aux pertes. ${ }^{25}$ Ils se tournent donc vers la loterie positive c'est-à-dire vers le mécanisme de rémunération contingente. Les auteurs ont ensuite mené plusieurs expérimentations qui confirment ce résultat, quels que soient le niveau de richesse des individus et le montant des coûts du système juridique.

De manière générale, suivant de cette littérature, l'honoraire de résultat est susceptible de faciliter l'accès au droit selon deux canaux de transmission : via un canal purement financier lui permettant de se substituer au mécanismes alternatifs de financement externe (aide juridictionnelle, financement par un tiers, ...) ou via un canal plus comportemental en constituant un mécanisme de régulation du phénomène d'aléa moral ou de transfert de risque, ainsi source d'ef-

23. Les auteurs rappellent néanmoins que ces arguments sont à relativiser. Du point de vue assurantiel, les avocats sélectionnent les affaires qui ne présentent pas de réels risques et refusent les autres. Du point du vue du crédit, les avocats n'encourent pas de risque puisqu'ils déduisent leurs honoraires avant de reverser ce qui leur revient à leur client.

24. Les auteurs font l'hypothèse que le point de référence correspond à la situation une fois le préjudice subi. Estimer que le point de référence correspond à la situation avant le dommage reviendrait à considérer que les loteries sont uniquement composées de pertes. S'appuyant sur la littérature en économie expérimentale, les auteurs écartent cette possibilité car les individus adaptent rapidement leur point de référence, de sorte qu'entre le moment où le dommage a lieu et le moment où le type d'honoraire est choisi, le demandeur a déjà modifié son point de référence.

25. L'aversion aux pertes est un biais comportemental impliquant que l'individu est plus sensible, en termes de bien-être, à une perte qu'à un gain de montant identique. 
ficience dans la relation avocat-client.

3.1.4. Honoraire contingent et droits de la défense. L'honoraire contingent serait-il susceptible de faciliter l'accès au droit des défendeurs? Cette question reste normative dans la mesure où les avocats des défendeurs sont très largement payés à l'heure ou au forfait aux Etats-Unis. Selon Dana et Spier (1993), cette différence ne se justifie cependant pas au regard des arguments portant sur le transfert de risque ou sur le contrôle de l'effort de l'avocat qui sont similaires pour les deux types de justiciables. Les raisons sont tout autres. D'une part, il apparaîtrait complexe de se référer à un cadre standard : le montant des dommages-intérêts demandés est fixé par le demandeur qui peut exagérer sa demande, ce qui pose un problème pour estimer les honoraires du défendeur sur cette base. D'autre part, la solvabilité du défendeur est généralement plus importante que celle du demandeur, dans la mesure où il s'agit souvent d'une firme couverte par un mécanisme d'assurance, ce qui rend le recours à l'honoraire de résultat moins pertinent. Enfin, il faut noter que le défendeur ne choisit pas d'aller en justice mais y est contraint par le demandeur. Dana et Spier considèrent néanmoins que l'introduction d'un honoraire contingent pour la défense pourrait avoir une certaine cohérence en permettant une meilleure protection de ses droits : un avocat payé au résultat incite le défendeur à accepter un arrangement uniquement s'il anticipe un mauvais jugement. Les résultats de Emons et Fluet (2016) sont plus nuancés. Ils cherchent à fournir un fondement théorique au fait stylisé mentionné, à savoir le fait que les avocats de la défense sont généralement payés sur une base forfaitaire tandis que ceux des demandeurs le sont au résultat. L'argument est le suivant : la rémunération fixe de l'avocat du défendeur, qui lui garantit qu'il sera défendu quel que soit son risque de perdre le procès, couplée à une rémunération contingente de l'avocat du demandeur, qui est incité à conseiller à son client d'intenter des poursuites uniquement si l'affaire est solide (puisqu'il anticipe que le défendeur sera toujours défendu par un avocat), protège dans une certaine mesure les droits du défendeur. Zamir et Ritov (2010), dans leur étude expérimentale, ont apporté une explication complémentaire au choix du type d'honoraire par les défendeurs. Par rapport à leur situation de référence, les deux types d'honoraire constituent des pertes pour les défendeurs mais l'intervalle des pertes est plus large sous l'honoraire forfaitaire que sous l'honoraire contingent : dans le premier cas, le défendeur paye le montant des honoraires s'il gagne ou ce montant auquel s'ajoutent les dommages-intérêts s'il perd; dans le second cas, le défendeur ne paye rien s'il gagne et paye le montant du dommage s'il perd. Les auteurs observent alors que les défendeurs optent majoritairement pour l'honoraire forfaitaire, manifestant une préférence pour le risque en cas de pertes (suivant en cela le concept d'aversion aux pertes évoqué en amont).

\subsection{Qualité des recours en justice}

Le type de rémunération des avocats est depuis longtemps au coeur de débats concernant le nombre et la qualité (ou le mérite) des affaires portées en justice, certains modes de rémunération étant accusés d'encourager le développement d'affaires de faible qualité. La qualité d'une affaire s'apprécie au regard de plusieurs éléments potentiels, tels que la réalité ou l'absence du préjudice, la probabilité de victoire, ou encore la valeur du jugement en cas de victoire. Selon ces critères, on peut ainsi distinguer les affaires selon leur qualité élevée ou faible, celles relevant de la seconde 
catégorie étant qualifiées par la littérature de "recours opportunistes" (frivolous suits ou nuisance suits). La définition de tels recours apparaît cependant large, cette notion recouvrant en réalité plusieurs définitions, comme le note Bone (1997), qu'il parait utile d'exposer dans la perspective du lien entre ce type d'affaires et le mode de rémunération des avocats. La première définition considère un recours comme opportuniste si la valeur espérée de l'affaire est négative, c'est-àdire si le jugement espéré en cas de procès est inférieur aux coûts juridiques supportés par le demandeur. ${ }^{26}$ Cependant, cette définition est relativement restrictive, puisque des coûts juridiques particulièrement élevés peuvent anéantir la valeur espérée de l'affaire alors même que le jugement attendu est élevé. Une seconde définition s'attache uniquement à la probabilité de victoire, en considérant un recours comme opportuniste dès lors que cette probabilité est très faible. Enfin, une troisième définition tient à la croyance du demandeur que sa probabilité de gagner est très faible. Un recours peut, à cet égard, être qualifié d'opportuniste si le demandeur est pessimiste, alors même que la probabilité objective est élevée. Quelle que soit la définition retenue, un demandeur opportuniste devrait préférer abandonner les poursuites plutôt que d'aller jusqu'à la phase du procès. Cependant, dans la mesure où le défendeur peut craindre des frais judiciaires élevés en cas de procès ou manquer d'informations sur le sérieux de la plainte, ce dernier pourrait faire une offre lors de la phase de négociation avant procès, justifiant ainsi le recours opportuniste.

Le débat portant sur le lien entre mode de rémunération et survenance de recours opportunistes s'est largement focalisé sur la comparaison entre rémunération fixe ou horaire et rémunération contingente, à l'exception de quelques articles portant sur la comparaison entre rémunération conditionnelle et autres modes de rémunération.

3.2.1. Honoraire contingent et sélection des affaires. Le point de départ de ce débat réside dans une assertion relativement courante, mais pourtant peu étayée par la littérature économique, selon laquelle la rémunération contingente des avocats serait une invitation à des recours opportunistes, dans la mesure où les demandeurs ne supportent aucun risque financier lié au fait d'intenter une action. En effet, avec ce type de rémunération comme avec tout autre mode n'impliquant ni paiement à l'heure, ni paiement fixe, le demandeur n'a aucun frais à supporter en cas de défaite, ce qui n'est pas de nature à l'amener à s'auto-sélectionner lorsqu'il intente une action. Les arguments en défaveur de ce mode de rémunération proviennent pour l'essentiel de juristes. Ainsi, par exemple, McKinnon (1964) est l'un des premiers à soutenir que l'hostilité envers la rémunération contingent serait due à plusieurs facteurs, et notamment le fait qu'en donnant à l'avocat le droit de financer un litige, celle-ci lui permettrait de financer des recours "nuisibles", c'est-à-dire avec un mérite faible. Ce point est d'ailleurs l'un des arguments ayant justifié le choix historique de certains pays, tels que l'Angleterre et le Canada, d'interdire l'honoraire contingent. Les critiques à l'encontre de la rémunération contingente sont pour certaines particulièrement acerbes. Ainsi, Bernstein (1996) estime que "la rémunération contingente encouragerait les avocats à s'engager dans des litiges de façon excessive dans l'espoir de décrocher occasionnellement un jackpot conséquent" (p.79). Cependant, très peu de travaux en réalité obtiennent des résultats en ce sens.

26. Le jugement espéré du procès est défini comme étant le montant des dommages-intérêts fixé par le juge pondéré par la probabilité de victoire. 
Les travaux théoriques en la matière convergent en effet en faveur de la rémunération contingente, à de rares exceptions près. Un premier argument est ainsi soulevé par Clermont et Currivan (1978), qui soutiennent que dans la mesure où la rémunération contingente transfère le risque du client vers l'avocat, ce dernier a une incitation à éviter les affaires ayant de faibles chances de succès. De surcroît, l'expertise de l'avocat le rendrait mieux à même de filtrer les affaires qu'un demandeur.

De même, Dana et Spier (1993) recherchent théoriquement le mode de rémunération optimal (au sens de l'intérêt du client), en comparant honoraires fixe et contingent, lorsque l'avocat est mieux informé que son client sur la qualité de l'affaire, appréhendée ici par la probabilité de victoire. Selon leur modèle, l'avocat rémunéré de façon fixe ou horaire a peu d'incitations à révéler à son client que son affaire a un rendement espéré faible. Quel que soit le mérite d'une affaire donnée, l'avocat pourrait ainsi être incité à emmener son client le plus loin possible dans le litige, réduisant ainsi le taux d'arrangement, comme nous l'avons mentionné dans la section 2.1. A l'inverse, l'avocat percevant une part du montant obtenu par son client, via la négociation ou le procès, aurait intérêt à agir dans l'intérêt de son client et à ne défendre que les affaires ayant un rendement espéré relativement élevé. Selon cet argument, en alignant les intérêts de l'avocat et de son client, la rémunération contingente serait donc la mieux à même de sélectionner les affaires de meilleure qualité et de réduire la proportion de recours qualifiés par les auteurs d'opportunistes (suivant la définition fournie en amont).

D'autres articles font l'hypothèse que le client est la partie informée, et non l'avocat. C'est le cas de Miceli (1994), dont la question de recherche est proche de celle de l'article précédent, son objectif étant de déterminer si la rémunération contingente est à l'origine d'un nombre excessif de litiges. Dans ce modèle, le client prend seul les décisions d'intenter une action et d'accepter ou de rejeter l'offre amiable du défendeur. La partie non informée est le défendeur, qui ignore le caractère fondé ou infondé de l'action du demandeur. En effet, le modèle se caractérise par la présence de demandeurs ayant réellement subi un préjudice ("vraies victimes") et de demandeurs opportunistes n'ayant pas subi de préjudice ("non-victimes" ou "fausses victimes"). Bien que le gain espéré des fausses victimes au procès soit de zéro, le défendeur n'est pas capable ex-ante de distinguer les vraies victimes des fausses; l'avocat ne peut pas non plus observer le type de la victime avant d'avoir accepté de défendre l'affaire. Une fausse victime peut donc avoir intérêt à intenter initialement une action dans le but d'extorquer un arrangement au défendeur. ${ }^{27} \mathrm{Ce}$ dernier fait alors une offre à prendre ou à laisser au demandeur, en fonction de son estimation de la probabilité a posteriori que le plaignant qui porte plainte soit une vraie victime; à l'équilibre, cette probabilité dépend à la fois de la proportion de vraies victimes dans la population et de la proportion de non-victimes qui portent plainte. Le résultat majeur du modèle réside dans le fait qu'une proportion plus importante de non-victimes dans la population totale influence négativement la décision du défendeur de faire une offre, ce qui au final réduit la part de nonvictimes qui intentent une action à l'équilibre. Ce résultat s'explique par le fait qu'en cas de rémunération contingente, le demandeur ne reçoit pas dans son intégralité l'offre qui lui est faite

27. Cet argument portant sur les recours opportunistes a été soulevé au départ par Bebchuk (1988) : la crédibilité de la menace du demandeur d'aller en justice serait fondée sur la présence d'une asymétrie d'information. 
par le défendeur. Ainsi, l'offre qui serait acceptée par un demandeur réellement victime est plus élevée que sous une rémunération horaire, ce qui réduit, toutes choses égales par ailleurs, la propension du défendeur à trouver un arrangement. Le taux d'arrangement est donc plus faible en rémunération contingente qu'en rémunération horaire, ce qui réduirait les incitations des nonvictimes à porter plainte.

Rubinfeld et Scotchmer (1993) analysent différents arrangements contractuels possibles en termes de rémunération en adoptant un cadre principal-agent, dans lequel le client est mieux informé que son avocat sur la qualité de son affaire tandis que l'avocat est mieux informé que son client sur ses propres compétences. Dans ce contexte, ils montrent que la rémunération contingente peut permettre aux clients de signaler la qualité de leur affaire et aux avocats de signaler leur compétence. ${ }^{28}$ L'idée sous-jacente est la suivante : un client bien informé disposant d'une affaire de qualité est prêt à payer une rémunération fixe relativement élevée et un pourcentage contingent relativement faible, et inversement pour un client ayant une affaire faible. De son côté, un avocat bien informé et compétent signale cette caractéristique en travaillant pour un pourcentage de rémunération relativement élevé. De ce point de vue, le fait de plafonner la rémunération contingente serait de nature à réduire le nombre d'affaires de mauvaise qualité (ainsi que le nombre d'affaires défendues par des avocats compétents).

L'article de Emons et Fluet (2016), évoqué en amont, s'éloigne des articles précédents dans la mesure où leur question de recherche a pour objectif de fournir un fondement théorique à un fait stylisé, à savoir que les avocats de la défense sont payés selon une rémunération fixe tandis que ceux de l'accusation sont rémunérés selon une rémunération contingente aux Etats-Unis. Dans leur modèle, l'avocat est mieux informé que le client sur la qualité des affaires, la première conséquence étant que ce sont les avocats qui décident de poursuivre ou d'abandonner une affaire. Ainsi, sous une rémunération fixe excédant les coûts marginaux, un avocat de la défense accepte toutes les affaires quelle que soit leur qualité, et est incité à toutes les poursuivre jusqu'au procès. Cela amène donc les avocats des demandeurs à poursuivre uniquement les affaires de bonne qualité et à abandonner celles de piètre qualité.

Le seul article à notre connaissance plaidant catégoriquement en défaveur de la rémunération contingente eu égard aux recours opportunistes est celui de Chen (2006). Ce dernier part du constat qu'un demandeur opportuniste ne peut avoir de chance d'obtenir un arrangement amiable que si son action en justice est crédible. Or, la littérature a identifié plusieurs mécanismes qui rendent une action crédible, tels que le fait que les coûts de litige soient des coûts irrécouvrables, le fait que le demandeur obtienne des paiements non-monétaires du fait d'aller au procès (Rasmusen, 1994) ou encore le fait que les coûts de litige soient étalés dans le temps (Bebchuk, 1996). Chen (2006) suggère un autre mécanisme, consistant précisément en l'utilisation d'une rémunération contingente. Dans ce modèle, le demandeur, neutre au risque, décide d'intenter ou non une action, puis fait une offre à prendre ou à laisser au défendeur s'il poursuit. Si le défendeur rejette cette offre, le demandeur décide alors d'aller ou non au procès. Sous une rémunération fixe, le demandeur n'a jamais intérêt à aller jusqu'au procès puisque le jugement espéré est négatif, de sorte qu'à l'équilibre, le défendeur n'accepte pas son offre. En revanche, sous une rémunération

28. Le service juridique peut en effet être vu comme un bien de crédence (voir Emons, 2001). 
contingente, le demandeur ne supportant aucun coût du fait d'aller au procès, la menace d'aller à cette étape devient crédible dans la mesure où il est le seul à prendre cette décision (et non son avocat), ce qui incite le défendeur à accepter son offre d'arrangement. Les résultats de cette analyse sont cependant à relativiser : d'une part, tout est connaissance commune entre les protagonistes et d'autre part, l'avocat n'a aucun pouvoir décisionnaire, menant à une issue dans laquelle un demandeur opportuniste choisirait d'aller en procès même si l'espérance de jugement était infiniment négative.

A notre connaissance, un seul article empirique a eu pour objectif de contribuer à ce débat portant sur le lien entre qualité des affaires et mode de rémunération des avocats. Helland et Tabarrok (2003) utilisent ainsi la méthode différence-en-différences pour tester la "qualité juridique", une qualité faible étant associée à une forte probabilité que le demandeur abandonne l'affaire sans offre amiable ni jugement, en fonction du type de rémunération (horaire ou contingente). Les auteurs comparent les données de deux groupes de 8 états fédérés américains : le premier groupe correspond à des états dans lesquels le pourcentage de rémunération contingente que les avocats peuvent demander est limité dans le domaine médical, mais pas dans le domaine automobile. Le second groupe correspond à des états qui n'imposent aucune limitation à ce pourcentage quel que soit le domaine. Selon les auteurs, la restriction dans l'usage de la rémunération contingente peut avoir un effet pervers. Leur argument est que certains frais tels que les frais de déposition ou de reprographie par exemple sont non-contingents et s'apparentent donc à des frais horaires : si les avocats perçoivent une rémunération contingente non plafonnée, ils renoncent généralement à tarifer ces frais divers. En revanche, si la rémunération contingente est limitée, alors ces frais seront répercutés sur le client et en conséquence, une plus grande proportion de la rémunération de l'avocat sera non-contingente. Ainsi, il serait selon eux peu probable que les restrictions sur la rémunération contingente réduisent la rémunération espérée des avocats : en effet, la compensation espérée pourrait rester constante, mais dans la mesure où la rémunération contingente est plafonnée, la rémunération horaire augmenterait, ce qui réduirait les incitations des avocats à filtrer les affaires. Les résultats de leur analyse montrent tout d'abord que le taux d'abandon d'actions en justice pour faute médicale est significativement bien plus élevé dans les états avec rémunération horaire que dans ceux avec rémunération contingente, alors qu'il n'existe pas de différences dans les affaires automobiles, ce qui s'expliquerait par l'absence de filtrage en amont. Par ailleurs, le délai d'obtention d'un accord est également plus élevé en rémunération horaire qu'en rémunération fixe. Ainsi, d'un point de vue global, cette analyse tendrait à démontrer que la rémunération horaire génèrerait des affaires de qualité plus faible que la rémunération contingente.

3.2.2. Honoraire conditionnel et sélection des affaires. Le premier article portant sur la rémunération conditionnelle est celui de Emons (2007), qui compare les rémunérations conditionnelle et contingente dans un cadre d'asymétrie d'information entre avocat et client sur la qualité de l'affaire, asymétrie au détriment de l'avocat. L'auteur détermine le mode de rémunération que doit offrir l'avocat à l'équilibre selon l'élément sur lequel porte cette asymétrie, à savoir le jugement espéré ou le risque associé à l'affaire. Si celle-ci porte sur le jugement espéré, les avocats doivent offrir une rémunération conditionnelle. En effet, si les clients ont le choix entre les deux types de rémunération, les clients ayant une affaire de qualité élevée opteraient pour une rémunération 
conditionnelle afin de ne pas partager les bénéfices résiduels. A l'inverse, les clients avec de faibles affaires opteraient pour une rémunération contingente puisque la part de l'avocat serait alors plus faible que sous une rémunération conditionnelle. Ainsi, dans ce cas, un avocat offrant une rémunération contingente n'attirerait que des affaires présentant un mérite faible, générant un phénomène de sélection adverse. En revanche, si l'asymétrie d'information porte sur le risque associé à l'affaire, alors la rémunération contingente prévaut à l'équilibre. En effet, en termes de rendement espéré tout d'abord, sous l'hypothèse qu'un risque élevé est associé à un enjeu du litige élevé, et un risque faible à un enjeu faible, si l'avocat propose un honoraire conditionnel, il obtient seulement un montant fixe en cas de victoire. Il n'obtient donc qu'une faible proportion du jugement si celui-ci est élevé, impliquant un rendement espéré des avocats décroissant avec le risque. A l'inverse, en honoraire contingent, l'avocat, qui obtient un pourcentage du jugement, est compensé d'une faible probabilité de victoire par une récompense élevée en cas de victoire. En outre, les clients à faible risque préfèrent l'honoraire contingent parce qu'une fraction du jugement restera certainement inférieure à l'honoraire conditionnel qu'ils auraient à payer. Ainsi, si l'avocat offrait un honoraire conditionnel, il attirerait seulement les clients à risque élevé, ce qui impliquerait une sélection négative des affaires; il a donc tout intérêt dans le cas d'une asymétrie sur le risque de l'affaire à opter pour un honoraire contingent. En effet, dans ce cas, si les deux rémunérations sont proposées au client, les clients à haut risque préfèrent la rémunération conditionnelle parce que les rendements espérés de l'avocats sont décroissants par rapport au risque. A l'inverse, les clients à faible risque préfèrent la rémunération contingente parce que pour eux, une fraction donnée du résultat est plus faible que la rémunération conditionnelle qu'ils auraient probablement à payer. Ainsi, un avocat offrant un contrat conditionnel n'attirerait que des clients à haut risque.

L'analyse de Gabuthy et Lambert (2011) s'intéresse également à la rémunération conditionnelle et leur objectif est proche de celui de Miceli (1994) qui compare rémunérations contingente et fixe. Ils s'éloignent cependant de Miceli sur deux points. D'une part, ils comparent rémunération conditionnelle et rémunération fixe. D'autre part, afin d'être cohérents avec le contexte de la rémunération conditionnelle qui caractérise le système juridique anglais, ils situent ce mode de rémunération dans le contexte d'une règle anglaise d'allocation des coûts plutôt qu'une règle américaine, cette dernière s'appliquant à la rémunération contingente. ${ }^{29}$ Dans le cadre d'un jeu en information asymétrique (dans lequel le défendeur ignore si le demandeur a véritablement subi un préjudice), les auteurs montrent que le système conditionnel améliore l'efficacité du processus en réduisant le nombre de recours opportunistes. L'explication du résultat est la suivante : comparé à une rémunération horaire, le bonus payé à l'avocat en cas d'arrangement accroît le montant nécessaire pour inciter un demandeur à conclure une transaction. Le montant amiable que le défendeur doit payer sous une rémunération conditionnelle est alors plus élevé que sous une rémunération horaire, ce qui le rend moins propice à trouver un arrangement et ainsi, réduit le rendement espéré d'un recours pour un demandeur opportuniste. En conséquence, en cas de rémunération conditionnelle, moins de demandeurs non-victimes intentent une action en justice.

29. La règle américaine stipule que chaque partie supporte ses propres coûts de procès, alors que la règle anglaise fait supporter l'intégralité des coûts à la partie perdante. 


\subsection{Efforts de précaution et survenance d'un accident}

Nous avons mis en relief dans les sections précédentes que les différents modes de rémunération des avocats peuvent influencer les incitations à poursuivre de la part des demandeurs ainsi que les stratégies mises en oeuvre par les différentes parties prenantes lors du règlement amiable du litige et/ou lors du procès éventuel. Forts de ce constat, il est clair que ces différents modes de rémunération sont également susceptibles d'avoir un impact en amont de la procédure judiciaire : étant donné leurs conséquences éventuelles, les mécanismes de rémunération des avocats peuvent influencer les efforts de précaution entrepris par les auteurs potentiels de dommages pour éviter la survenance d'un sinistre, et ainsi constituer une source d'efficience via leur impact potentiellement négatif sur le coût social des accidents.

Miceli et Segerson (1991) sont les premiers à prendre en compte cette influence indirecte de la rémunération des avocats sur les efforts de précaution. Dans un cadre de responsabilité avec une règle stricte, les auteurs comparent les implications de deux types de rémunération (horaire et contingente) en termes d'incitation du demandeur à poursuivre en justice et du défendeur à exercer un effort de précaution. ${ }^{30}$ Dans leur modèle, la valeur du préjudice subi constitue une information privée du demandeur, l'avocat et le défendeur ne connaissant que sa distribution. Le défendeur choisit un niveau de précaution de façon à minimiser sa perte espérée, sachant que ces précautions réduisent la probabilité de survenance d'un dommage, mais pas son ampleur. Deux systèmes de rémunération de l'avocat sont envisagés : dans un système de rémunération horaire, le demandeur supporte lui-même les coûts du procès et décide de poursuivre dès lors que la valeur du jugement attendu excède ces coûts. Par conséquent, les victimes se trouvant en queue de distribution dans les valeurs des jugements possibles ne portent pas leur affaire en justice. En cas de rémunération contingente, suivant l'hypothèse standard, les coûts de procès sont supportés par l'avocat qui reçoit une fraction du jugement. Cependant, l'avocat a toujours intérêt à accepter l'affaire sous ce mode de rémunération dans la mesure où il n'observe pas le type de son client avant d'avoir encouru les frais de procès. ${ }^{31}$ Ainsi, suivant ce schéma de rémunération, la victime est toujours incitée à poursuivre le défendeur en justice, la seule condition étant que la fraction qu'elle reçoit du jugement soit positive. En conséquence, la probabilité de procès conditionnelle à la survenance d'un accident est plus élevée sous une rémunération contingente que sous une rémunération horaire. Le défendeur anticipant cette probabilité de poursuites plus élevée à l'équilibre choisit un niveau de précaution plus important avec rémunération contingente qu'avec rémunération horaire. Globalement, l'effet du mode de rémunération sur le coût social des accidents est ambigu : comparativement à l'honoraire fixe ou horaire, l'honoraire contingent accroît, d'une part, la probabilité de poursuites conditionnelle à la survenance d'un accident mais diminue, d'autre part, la probabilité d'accident via son effet positif sur l'effort de précaution. Le modèle étendu à une règle de responsabilité pour faute mène à des conclusions plus nuancées :

30. La règle de responsabilité stricte (ou sans faute) implique que l'auteur du dommage est considéré comme responsable et doit ainsi indemniser la victime ex-post, quel que soit le niveau de précaution choisi ex-ante.

31. Le marché des avocats étant supposé concurrentiel et la victime ne choisissant pas le mode de rémunération, les profits espérés des avocats sont nuls, de sorte que les surplus espérés sur les procès rentables compensent les pertes espérées sur les procès non rentables. 
selon qu'un type de rémunération amène l'auteur du dommage à satisfaire ou non le standard, les incitations en termes de poursuites et le volume de procès peuvent différer des résultats obtenus avec une règles de responsabilité stricte. ${ }^{32}$

L'analyse de Gravelle et Waterson (1993) complète celle de Miceli et Segerson (1991) notamment sur deux points : d'une part, leur modèle intègre une phase supplémentaire qui est celle de l'arrangement pré-procès, et d'autre part, ils proposent une comparaison de deux règles d'allocation des coûts de procès : la règle américaine, selon laquelle chaque partie supporte ses propres coûts de procès, et la règle anglaise, selon laquelle la partie perdante doit assumer ses propres coûts ainsi que ceux de la partie gagnante. Ainsi, suivant Miceli et Segerson (1991), le défendeur peut réduire la probabilité de survenance d'un accident par la prise de précautions mais est toujours poursuivi en cas d'accident. En outre, dans le cas de poursuites, ce dernier fait au demandeur une offre d'arrangement à prendre ou à laisser, sachant qu'un refus de l'offre implique la tenue d'un procès. La décision d'accepter ou non cette offre est prise par l'avocat du demandeur, qui pondère son propre gain et celui de son client selon un paramètre d'altruisme dont la valeur est connue du défendeur mais non observée par le client. Dans le modèle de base, l'honoraire de résultat implique le versement d'une prime fixe à l'avocat, par le demandeur, dont le montant diffère selon qu'il y a arrangement ou procès et dans ce cas, selon que le procès est perdu ou remporté. Par ailleurs, l'avocat est supposé assumer seul les coûts du procès. Dans ce contexte, Gravelle et Waterson (1993) montrent que le seuil d'acceptation de l'offre par l'avocat (défini comme la valeur du jugement en deçà de laquelle l'avocat accepte l'offre de règlement amiable) dépend des caractéristiques du contrat entre ce dernier et son client, ainsi que du paramètre d'altruisme. Ce seuil peut être utilisé ex-ante par le défendeur dans le choix de son offre d'arrangement, et de ce fait influencer la probabilité d'accord de par l'impact à la fois sur l'offre du défendeur et sur la propension à accepter une offre donnée. Enfin, les auteurs montrent que les caractéristiques du contrat et l'altruisme de l'avocat peuvent accroître le niveau de précaution du défendeur et ainsi réduire la probabilité d'accident s'ils diminuent le seuil d'acceptation de l'offre. Le modèle de base est ensuite étendu à trois modes de rémunération : un contrat qualifié de "normal" dans lequel l'avocat reçoit une rémunération fixe égale à ses coûts, un contrat avec honoraire conditionnel (où l'avocat reçoit un paiement égal à ses coûts auxquels s'ajoute un bonus en cas d'arrangement ou de victoire), et enfin un contrat fondé sur une rémunération contingente (où l'avocat reçoit une part du jugement en cas de victoire et ne reçoit rien en cas de défaite). Les auteurs montrent notamment que la probabilité d'arrangement est plus forte et la probabilité d'accident plus faible avec les deux derniers types de contrats si l'avocat est plutôt égoïste, c'est-à-dire pondère davantage son gain que celui de son client. En effet, ces contrats réduisent la propension de son client à accepter une offre donnée, mais augmentent son propre gain.

Lambert et Chappe (2014) prennent également en compte l'influence du mode de rémunération de l'avocat sur les incitations du défendeur à prendre des précautions et sur celles du demandeur à poursuivre en justice, mais leur objectif est avant tout de comparer les effets de l'honoraire de résultat (contingent et conditionnel) à ceux de l'aide juridictionnelle. Cette dernière est modélisée

32. Dans un régime de responsabilité pour faute, l'auteur du dommage est reconnu responsable uniquement si son niveau de précaution est inférieur à une valeur standard (définie par le juge ou par la loi). 
alternativement comme une aide proportionnelle ou comme une aide forfaitaire et ayant une influence positive sur sa probabilité de victoire. Comme nous l'avons évoqué précédemment, les auteurs montrent que l'incitation du demandeur à poursuivre est plus forte avec un honoraire de résultat qu'avec un système d'aide juridictionnelle dans la mesure où dans le premier cas, le demandeur ne supporte aucun coût. Cependant, leur conclusion est plus nuancée quant à l'influence sur les incitations aux précautions dans la mesure où cette influence dépend de l'effet du mode de financement sur la probabilité de victoire du demandeur. Si cette probabilité de victoire est plus forte avec une rémunération contingente qu'avec l'aide juridictionnelle, alors les incitations du défendeur à prendre des précautions sont nécessairement plus fortes dans le premier cas puisqu'elles vont dans le même sens que l'incitation à poursuivre mentionnée précédemment. A l'inverse, si la probabilité de victoire est plus forte avec l'aide juridictionnelle qu'avec l'honoraire au résultat, alors l'issue est ambigue : si l'effet de l'aide juridictionnelle sur la probabilité de victoire l'emporte sur l'effet en termes d'incitations à poursuivre, alors les incitations aux précautions sont plus fortes avec aide juridictionnelle. En revanche, si l'effet en termes d'incitations à poursuivre fait plus que compenser l'effet sur la probabilité de victoire, alors l'aide juridictionnelle est moins incitative en termes de prise de précautions que l'honoraire de résultat.

\section{Conclusion}

Cet article a pour objet de faire le point sur une littérature aujourd'hui abondante relative à l'analyse des modalités de rémunération des avocats dans le cadre d'actions en responsabilité civile. A cet égard, cette littérature met en lumière le fait que l'honoraire de résultat (impliquant une rémunération à la performance) présenterait plusieurs avantages potentiels, notamment en termes d'accès au droit, de sélection des affaires, de transfert de risque et de régulation du phénomène d'aléa moral. Néanmoins, comme nous l'avons évoqué en introduction, les analyses sous-jacentes restent très majoritairement d'ordre théorique, limitant de fait la portée des éventuelles recommandations de politique publique que nous pourrions formuler. Or, d'un point de vue méthodologique, les moyens d'investigation empirique en la matière apparaissent limités, et ce pour deux raisons principales. D'une part, un degré de confidentialité important est souvent attaché à la question de la résolution des litiges, ce qui rend l'accès à des données de terrain particulièrement difficile. D'autre part, les moyens d'analyse empirique sont de fait complexes lorsque l'on souhaite observer le changement de comportement des individus suite à la mise en place d'une nouvelle législation. En effet, ce type de situation n'est observable qu'à la condition que certaines politiques soient mises en oeuvre. Cette restriction est particulièrement importante dans le domaine qui nous intéresse puisqu'il s'agit d'observer les modifications de comportement des parties prenantes au litige suite à la mise en oeuvre d'un nouveau système de rémunération des avocats, à savoir l'honoraire de résultat. A cet égard, la méthode expérimentale apparaît prometteuse dans la mesure où elle permet de simuler l'abstraction de la théorie dans le cadre contrôlé d'un laboratoire et ainsi de provoquer cette situation (afin d'en observer les conséquences sur les comportements individuels). Sur cette base, la méthode permet de s'assurer que ces comportements sont bien le fruit des changements de législation et non de causes tierces échappant à l'observation. Cette condition dite d' "exogénéité" est ainsi au cœur des difficultés rencontrées par l'analyse 
empirique de l'économie du droit. Dans son célèbre article consacré aux enjeux méthodologiques de l'expérimentation en économie, Roth (1988) évoque la capacité de cette méthode à parler à l'oreille des princes (whispering in the ears of princes), à savoir orienter et inspirer la conduite de politiques publiques. A cet égard, il nous semble que le recours à l'économie expérimentale serait d'un grand intérêt pour conseiller le législateur s'agissant de l'adoption de mécanismes alternatifs de rémunération des avocats en matière civile, et pourrait être le support d'un programme de recherche particulièrement intéressant pour l'économiste. 


\section{Références}

Aт, C., eт Y. Gabuthy (2015) : "Moral Hazard and Agency Relationship in Sequential Litigation," International Review of Law and Economics, 41, 86-90.

Baumann, F., et T. Friehe (2012) : "Contingent Fees Meet the British Rule : An Exploratory Study," Public Choice, 150(3), 499-510.

Bebchuk, L. A. (1988) : "Suing Solely to Extract a Settlement Offer," Journal of Legal Studies, $17(2), 437-450$.

(1996) : "A New Theory Concerning Credibility and Success of Threats to Sue," Journal of Legal Studies, 25(1), 1-25.

Bebchuk, L. A., et A. T. Guzman (1996) : "How Would You Like to Pay for That? The Strategic Effects of Fee Arrangements on Settlement Terms," Harvard Negotiation Law Review, $1,53-63$.

Bernstein, D. E. (1996) : "Procedural Tort Reform : Lessons from Other Nations," Regulation, 19(1), 71-81.

Bone, R. G. (1997) : "Modeling Frivolous Suits," University of Pennsylvania Law Review, 145(3), 529-605.

Bouquin, J.-P. (2004) : "L'assurance de protection juridique des particuliers," Discussion paper, Fédération Française des Sociétés d'Assurance.

Casagrande, A., et M. Spallone (2007) : "Investigating the Determinants of Pretrial Settlement Rates : Contingent versus Non-Contingent Lawyers' Fees," European Journal of Law and Economics, 24(1), 1-13.

Chambaz, C. (2017) : "Les chiffres-clés de la Justice 2017," Discussion paper, Ministère de la Justice.

Chappe, N., et Y. Gabuthy (2013) : "The Influence of Lawyers and Fee Arrangements on Arbitration," Journal of Institutional and Theoretical Economics, 169(4), 720-738.

Chen, Z. (2006) : "Nuisance Suits and Contingent Attorney Fees," Review of Law and Economics, $2(3), 363-370$.

Chor, A. (2003) : "Allocating Settlement Authority under a Contingent-Fee Arrangement," Journal of Legal Studies, 32(2), 585-610.

Clermont, K. M., et J. D. Currivan (1978) : "Improving on the Contingent Fee," Cornell Law Review, 63(4), 529-639.

Dana, J. D., Et K. E. SpIER (1993) : "Expertise and Contingent Fees : The Role of Asymmetric Information in Attorney Compensation," Journal of Law, Economics, ES Organization, 9(2), 349-367. 
Danzon, P. M. (1983) : "Contingent Fees for Personal Injury Litigation," Bell Journal of Economics, 14(1), 213-224.

Deffains, B., et C. Desrieux (2015) : "To Litigate or Not to Litigate? The Impacts of ThirdParty Financing on Litigation," International Review of Law and Economics, 43, 178-189.

Deffains, B., et E. Langlais (2009) : Analyse écononomique du droit. De Boeck.

Dover, M. A. (1986) : "Contingent Percentage Fees : An Economic Analysis," Journal of Air Law and Commerce, 51(2), 531-566.

Emons, W. (2001) : "Credence Goods Monopolists," International Journal of Industrial Organization, 19(3-4), 375-389.

(2007) : "Conditional versus Contingent Fees," Oxford Economic Papers, 59(1), 89-101.

Emons, W., et C. Fluet (2016) : "Why Plaintiff's Attorneys Use Contingent and Defense Attorneys Fixed Fee Contracts," International Review of Law and Economics, 47, 16-23.

Emons, W., et N. Garoupa (2006) : "US-style Contingent Fees and UK-style Conditional Fees : Agency Problems and the Supply of Legal Services," Managerial and Decision Economics, 27(5), $379-385$.

Farmer, A., et P. Pecorino (2005) : "Dispute Rates and Contingency Fees : An Analysis from the Signaling Model," Southern Economic Journal, 71(3), 566-581.

Faure, M., F. Fernhout, et N. J. Philipsen (2010) : "No Cure, No Pay and Contingency Fees," in New Trends in Financing Civil Litigation in Europe, ed. by M. Tuil, et L. Visscher. Edward Elgar.

Faure, M. G., Et J. P. D. Mot (2012) : "Comparing Third Party Financing of Litigation and Legal Expenses Insurance," Journal of Law, Economics and Policy, 8(3), 743-778.

Gabuthy, Y., et E.-A. Lambert (2011) : "English Rule and Frivolous Suits : Conditional versus Hourly Fees," Review of Law and Economics, 7(1), 75-88.

Garoupa, N., et F. Gomez-Pomar (2007) : "Cashing by the Hour : Why Large Law Firms Prefer Hourly Fees over Contingent Fees," Journal of Law, Economics, ES Organization, 24(2), $458-475$.

Gravelle, H., et M. Waterson (1993) : "No Win, No Fee : Some Economics of Contingent Legal Fees," Economic Journal, 103(420), 1205-1220.

HAdfield, G. K. (2000) : "The Price of Law : How the Market for Lawyers Distorts the Justice System," Michigan Law Review, 98(4), 953-1006.

Halpern, P. J., et S. M. Turnbull (1983) : "Legal Fees Contracts and Alternative Cost Rules : An Economic Analysis," International Review of Law and Economics, 3(1), 3-26. 
Hay, B. L. (1996) : "Contingent Fees and Agency Costs," Journal of Legal Studies, 25(2), 503533.

(1997) : "Optimal Contingent Fees in a World of Settlement," Journal of Legal Studies, 26(1), 259-278.

Helland, E., et A. Tabarrok (2003) : "Contingent Fees, Settlement Delay, and Low-Quality Litigation : Empirical Evidence from Two Datasets," Journal of Law, Economics, 8 Organization, 19(2), 517-542.

Hyde, C. E. (2006) : "Conditional versus Contingent Fees : Litigation Expenditure Incentives," International Review of Law and Economics, 26(2), 180-194.

Laffont, J.-J., et D. Martimort (2002) : The Theory of Incentives : The Principal-Agent Model. Princeton University Press.

Lambert, E.-A., et N. Chappe (2014) : "Litigation with Legal Aid versus Litigation with Contingent/Conditional Fees," Review of Law and Economics, 10(1), 95-115.

Landes, W. M. (1971) : "An Economic Analysis of the Courts," Journal of Law and Economics, $14(1), 61-107$.

(1993) : "Sequential versus Unitary Trials : An Economic Analysis," Journal of Legal Studies, 22(1), 99-134.

Leshem, S. (2009) : "Contingent Fees, Signaling and Settlement Authority," Review of Law and Economics, 5(1), 435-460.

McKee, M., R. Santore, et J. Shelton (2007) : "Contingent Fees, Moral Hazard, and Attorney Rents : A Laboratory Experiment," Journal of Legal Studies, 36(2).

McKinnon, F. B. (1964) : Contingent Fees for Legal Services. Chicago : Aldine Publishing Company.

Miceli, T. J. (1994) : "Do Contingent Fees Promote Excessive Litigation?," Journal of Legal Studies, 23(1), 211-224.

Miceli, T. J., et K. Segerson (1991) : "Contingent Fees for Lawyers : The Impact on Litigation and Accident Prevention," Journal of Legal Studies, 20(2), 381-399.

Miller, G. P. (1987) : "Some Agency Problems in Settlement," Journal of Legal Studies, 16(1), $189-215$

Perry, R. (2018) : "Crowdfunding Civil Justice," Boston College Law Review, 59(4), pp. 13571395.

Polinsky, A. M., Ex D. L. Rubinfeld (2002) : "A Note on Settlements under the Contingent Fee Method of Compensating Lawyers," International Review of Law and Economics, 22, 217225 . 
(2003) : "Aligning the Interests of Lawyers and Clients," American Law and Economics Review, 5(1), 165-188.

Posner, R. A. (1973) : "An Economic Approach to Legal Procedure and Judicial Administration," Journal of Legal Studies, 2(2), 399-458.

- (1996) : Law and Legal Theory in England and America. Oxford Clarendon Press.

Rasmusen, E. (1994) : Games and Information : An Introduction to Game Theory, 2nd edition. New York : Blackwell.

Reinganum, J. F., et L. L. Wilde (1986) : "Settlement, Litigation, and the Allocation of Litigation Costs," RAND Journal of Economics, 17(4), 557-566.

Rickman, N. (1999) : "Contingent Fees and Litigation Settlement," International Review of Law and Economics, 19, 295-317.

Roth, A. E. (1988) : "Laboratory Experimentation in Economics : A Methodological Overview," Economic Journal, 98(393), 974-1031.

Rubinfeld, D. L., et S. Scotchmer (1993) : "Contingent Fees for Attorneys : An Economic Analysis," RAND Journal of Economics, 24(3), 343-356.

- (1998) : "Contingent Fees," in The New Palgrave Dictionary of Economics and the Law, ed. by P. Newman, vol. 1. Mac Millan.

SPIER, K. E. (2007) : "Litigation," in Handbook in Law and Economics, ed. by A. M. Polinsky, et S. Shavell, vol. 1. Elsevier.

Thomason, T. (1991) : "Are Attorneys Paid What They're worth?," Journal of Legal Studies, 20(1), 187-223.

WAng, S. (2008) : "The Optimality of Contingent Fees in the Agency Problem of Litigation," International Review of Law and Economics, 28(1), 23-31.

Zamir, E., Et I. Ritov (2010) : "Revisiting the Debate over Attorneys' Contingent Fees : A Behavioral Analysis," Journal of Legal Studies, 39(1), 245-288. 